\title{
Thrust Balance Characterization of a 200W Quad Confinement Thruster for High Thrust Regimes
}

\author{
IEPC-2013-155 \\ Presented at the 33rd International Electric Propulsion Conference, \\ The George Washington University •Washington, D.C. •USA \\ October $6-10,2013$ \\ Aaron Knoll ${ }^{1}$, Dimitrios Lamprou ${ }^{2}$ and Vaios Lappas ${ }^{3}$ \\ University of Surrey, Guildford, Surrey, GU2 7XH, United Kingdom \\ Mark Pollard ${ }^{4}$ \\ Surrey Satellite Technology Ltd., Stephenson Road, Surrey Research Park, Guildford, GU2 7YE, United Kingdom \\ and \\ Paolo Bianco ${ }^{5}$ \\ Astrium Ltd., Anchorage Road, Portsmouth, Hampshire, PO3 5PU, United Kingdom
}

\begin{abstract}
A thrust balance characterization of a low powered Quad Confinement Thruster is presented for high levels of propellant flow. The nominal flow rate for this device is between $1 \mathrm{sccm}$ and $2 \mathrm{sccm}$ of Xenon propellant. This study extends the operating range, and investigates the performance at two high flow conditions of $10 \mathrm{sccm}$ and $20 \mathrm{sccm}$. Power is varied incrementally between $20 \mathrm{~W}$ and $200 \mathrm{~W}$ in order to characterize the performance versus power trends of the device. It was found that for these high flow regimes the propellant is underutilized, and a proportion of the increased thrust can likely be attributed to a hot gas expansion of the neutral Xenon rather than the generation of additional accelerated ions. The thrust was increased from $1 \mathrm{mN}$ (nominal) to $3.3 \mathrm{mN}$ at $200 \mathrm{~W}$ of input power for the $20 \mathrm{sccm}$ condition. However, the performance penalty in terms of the specific impulse was considerable. The specific impulse under these conditions dropped below 200s, where the nominal condition is 1000s. A compromise between increased thrust and decreased performance was found at $10 \mathrm{sccm}$ of flow: $3 \mathrm{mN}$ of thrust at 300s specific impulse.
\end{abstract}

\section{Nomenclature}

$B=$ magnetic field strength

$E \quad=\quad$ electric field strength

$F \quad=\quad$ force measured by the thrust stand

$g=$ gravity of earth

$l=$ length of the pendulum arm suspending the calibration weight

$m_{c}=$ mass of the calibration weight

$s \quad=$ horizontal displacement distance of the calibration weight

${ }^{1}$ Lecturer, Surrey Space Centre, email: a.knoll@ @urrey.ac.uk

${ }^{2}$ Research Student, Surrey Space Centre, email: d.lamprou@ surrey.ac.uk

${ }^{3}$ Professor, Surrey Space Centre, email: v.lappas@ surrey.ac.uk

${ }_{5}^{4}$ Senior Propulsion Engineer, Propulsion Group, email: m.pollard@ @stl.co.uk

5 ENS Electric Propulsion Engineering Team Leader, Electric Propulsion Department, email: paolo.bianco@astrium.eads.net 


\section{Introduction}

$\mathrm{T}$ HE Quad Confinement Thruster (QCT) is a new concept in electric propulsion with a key benefit of being able to vector the direction of thrust without the use of moving components. The principle of operation is similar to a Hall Effect Thruster (HET). A magnetic field is imposed between an anode and an external hollow cathode neutralizer. An electric field is sustained across the magnetic barrier, which acts to accelerate a beam of ions from the device. Thrust vectoring is accomplished by manipulating the topology of the imposed magnetic field barrier, which is formed by a quadrupole arrangement of eight electromagnets spaced around the periphery of the thruster. Previous experiments have shown a thrust vectoring capability of approximately 11 degrees off of the thruster axis (22 degree total angular range). ${ }^{1} \quad$ The magnetic topology for a symmetrically imposed field is illustrated in Fig. 1.

Although a relative newcomer in the field of electric propulsion (invented in 2009), the QCT has been receiving much commercial interest and support leading to a rapid progression of the technology. A joint program is currently underway

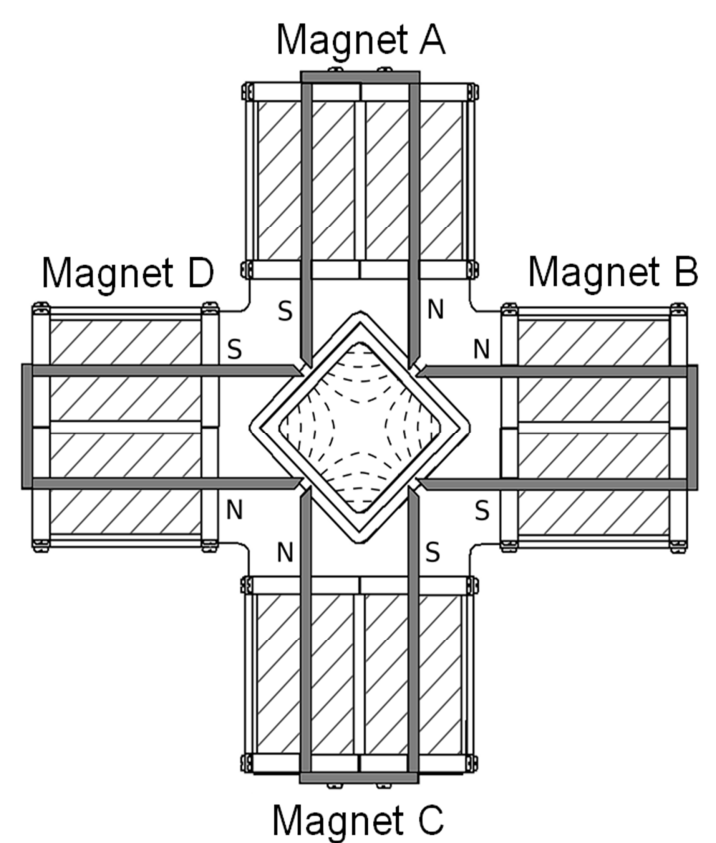

Figure 1. Magnetic field topology for the Quad Confinement Thruster.

between the Surrey Space Centre (SSC), Surrey Satellite Technology Ltd. (SSTL), and Astrium to design and qualify a flight version of the $200 \mathrm{~W}$ thruster. This paper presents the results of experimental thrust balance measurements of the 200W QCT device over an extended range of high flow rates. Previous thrust measurements have focused on optimizing the specific impulse of the device at low powers. ${ }^{2}$ The emphasis on achieving high specific impulse has steered previous experiments in the direction of low flow rates. However, a trade-off exists between specific impulse and the thrust to power ratio. During certain phases of a mission, satellite agility can be a higher priority than propellant utilization. This study considers higher mass flow rates aimed at maximizing the thrust to power ratio of the device. These experiments are conducted in the SSC propulsion test facility using a lab model design that closely matches the flight model QCT-200 under development at SSTL (see Fig. 2). The data gathered in this study provides valuable insight into the performance of the QCT operating in high flow regimes, and whether these modes of operation should be considered during a flight campaign.

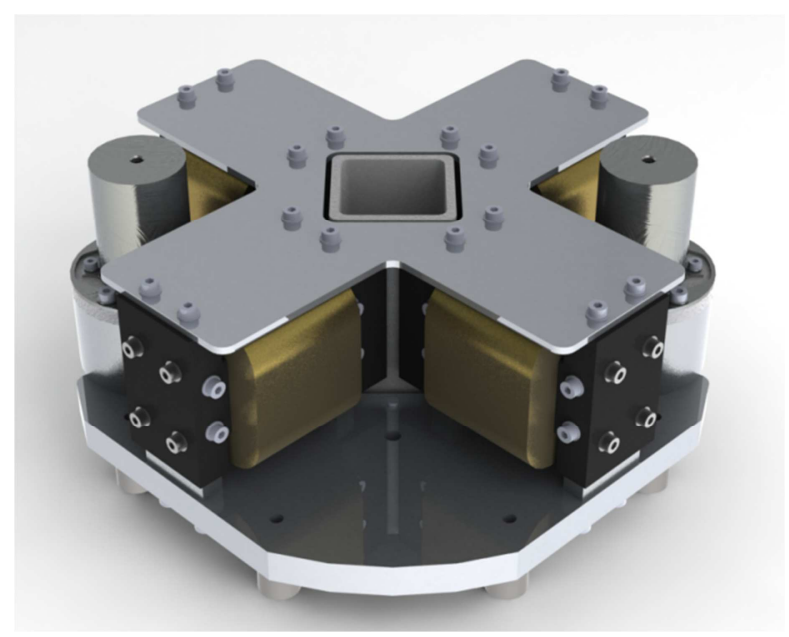

Figure 2. 200W Flight Model QCT-200. 


\section{Experimental Setup}

\section{A. Thruster}

The thruster investigated in this study is a $200 \mathrm{~W}$ lab model that was designed and constructed within the SSC. This device is referred to internally as the QCTII. A cut-away view of the QCT-II is shown in Fig. 3. The acceleration channel is constructed of Boron Nitride ceramic and has a square cross section with dimensions $35 \mathrm{~mm} \times 35 \mathrm{~mm} \times 55 \mathrm{~mm}$. The anode is made of Tantalum and has a square cross section with a $25 \mathrm{~mm}$ side length.

The magnetic field extends the full axial length of the acceleration channel, and is produced using eight electromagnets arranged into four quadrants as shown in Fig. 1. The topology of the magnetic field is essentially a quadrupole, with the field focused into four cusps using soft iron rails. Aluminum wire, rather than copper, was selected for the electromagnets in order to reduce the mass of the device. Since the strength of the magnetic field varies as a function of position within the channel, the magnetic field referenced in experiments refers to the magnitude as measured in the corner of the acceleration channel. For all of the experiments undertaken in this study, a magnetic field strength of 250 Gauss was used.

A hollow cathode is used to neutralize the outgoing ion beam and provide an electron current to sustain the discharge. The hollow cathode used for this study was developed and constructed internally within SSC, and is the same design as the hollow cathode being flown on the TechDemoSat (TDS) mission. ${ }^{3}$ Xenon is used as propellant for the QCT-II as well as the supply gas for the hollow cathode.

Unlike the Hall Effect Thruster, the ExB drift of electrons does not form a closed path. The directionality of the $\mathrm{E} \times \mathrm{B}$ drift is illustrated in Fig. 4. Electrons are drawn to the center of the channel along the horizontal plane, and expelled to the channel walls along the vertical plane. This brings both an advantage and disadvantage to the device. The disadvantage is that the open drift of electrons limits the efficiency of the thruster. However, because electrons are being drawn to the center of the channel where they have a clear path to the anode, the operating voltage tends to be significantly lower than other EP alternatives. The QCT typically runs at anode voltages lower than 70V. This is a significant advantage when considering the design of the power supply electronics because the anode voltage is

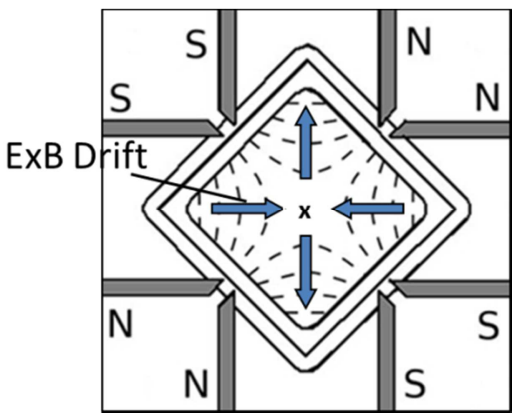

Figure 4. E×B electron drift orientation in the channel of the QCT. 


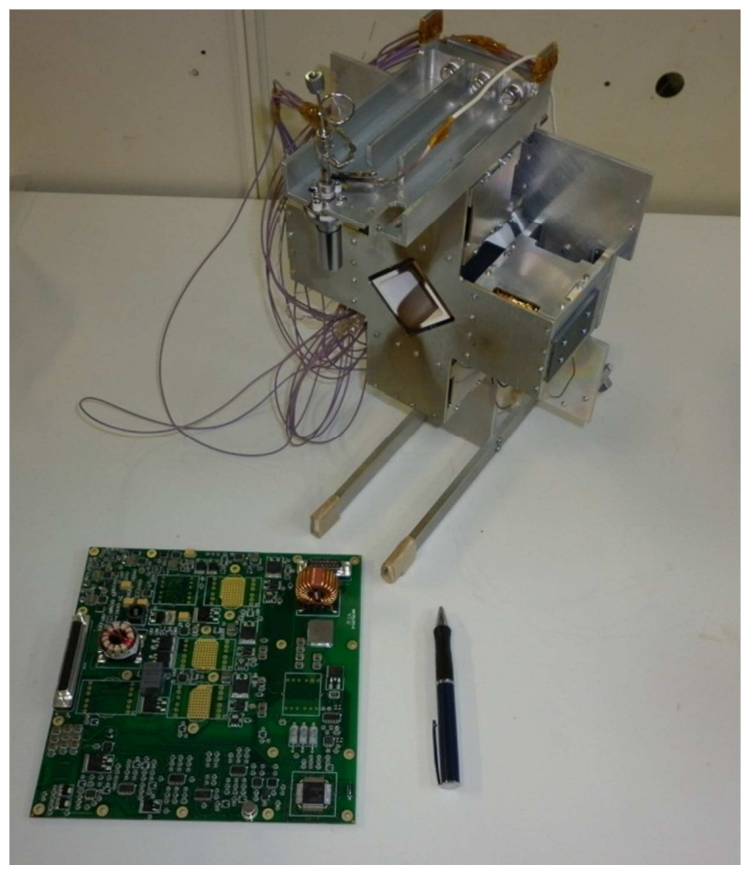

Figure 5. QCT-II plus power and control electronics board.

relatively close to the spacecraft bus voltage. The electronics can therefore be lighter, smaller, and more efficient. An Engineering Model (EM) version of the power supply and control electronics are shown alongside the QCT-II in Fig. 5. These electronics provide the power and control for the anode, electromagnets, and two redundant hollow cathodes.

\section{B. Thrust Stand and Vacuum Facility}

Thrust measurements were collected using the SSC thrust stand shown schematically in Fig. 6. This is an inverted pendulum design, which displaces horizontally under an applied force. The displacement is measured using a commercial laser displacement sensor (Micro-Epsilon model ILD 1700-2), which operates over a $2 \mathrm{~mm}$ range. The laser sensor is fixed to a rigid base while the laser target is suspended from the moving platform. The SSC thrust stand has been used for a number of previous experimental campaigns ${ }^{4}$, and has a precision of approximately $\pm 0.1 \mathrm{mN}$.

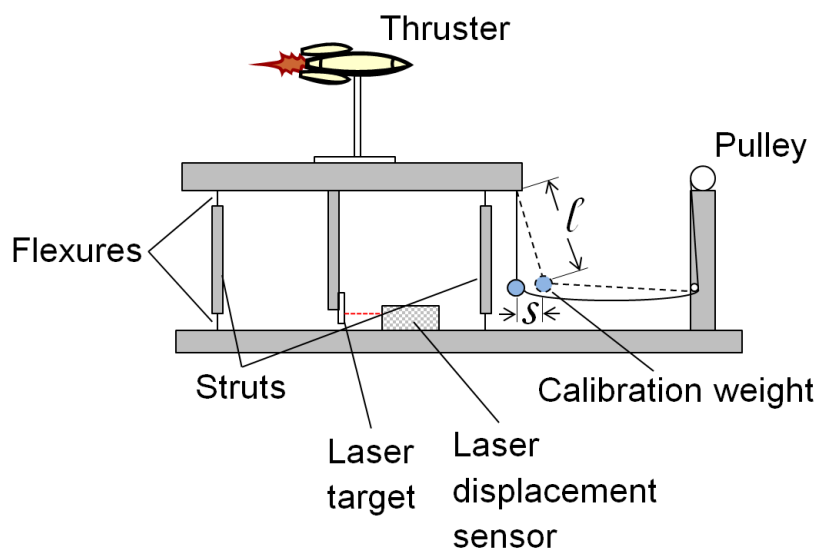

Figure 6. Schematic of the SSC thrust stand. 
The thrust stand is calibrated prior to each experiment by displacing a $5 \mathrm{~g}$ mass by a fixed distance. The horizontal force applied to the thrust stand can be determined geometrically by

$$
F=\frac{m_{c} g s}{\sqrt{l^{2}-s^{2}}},
$$

where $F$ is the horizontal component of force, $m$ is the mass of the calibration weight, $g$ is the gravity of earth, $s$ is the horizontal displacement distance of the calibration weight, and $l$ is the length of the pendulum thread suspending the weight.

The large SSC vacuum chamber (Daedalus) was used for these experiments. This is a cylindrical vessel with a diameter of $2.5 \mathrm{~m}$ and a length of $3.5 \mathrm{~m}$. This vacuum chamber uses two pumps operated in parallel: a cryogenic and turbomolecular pump with a combined capacity of 12,000 liters per second (air). The vacuum chamber reaches a pressure of mid $10^{-7}$ Torr without propellant flow, and mid $10^{-5}$ Torr while experiments are in progress.

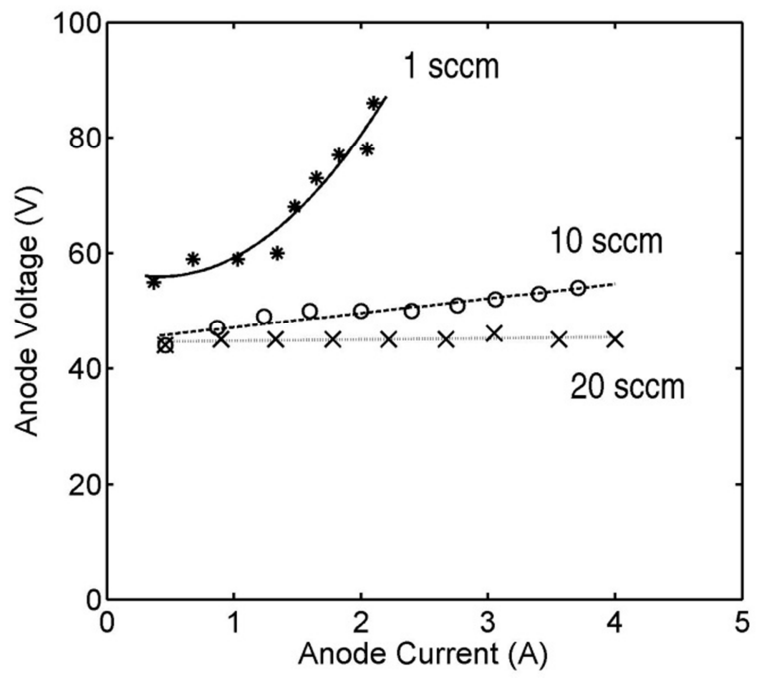

Figure 7. Anode voltage versus current at 1, 10, and 20 sccm flow rates.

\section{Results}

Three propellant flow rates were considered in this study: $1 \mathrm{sccm}, 10 \mathrm{sccm}$, and $20 \mathrm{sccm}$ of Xenon propellant. The $10 \mathrm{sccm}$ and $20 \mathrm{sccm}$ conditions represent significantly higher flow rates than previously investigated. Prior experiments have focused on flow rates up to $6 \mathrm{sccm}$, where the performance of the thruster is known to be higher. ${ }^{2}$ The $1 \mathrm{sccm}$ condition represents the baseline performance of the thruster, and was also included in this study in order to compare directly between the high and low flow regimes.

The current and voltage characteristics of the QCT are provided in Fig. 7 for the three flow conditions. For the $1 \mathrm{sccm}$ flow rate, the voltage increases in proportion to the square of the current. For the $10 \mathrm{sccm}$ flow rate there is a slow linear increase in voltage with increasing current, and for the $20 \mathrm{sccm}$ flow rate the voltage is constant for currents up to $4 \mathrm{~A}$.

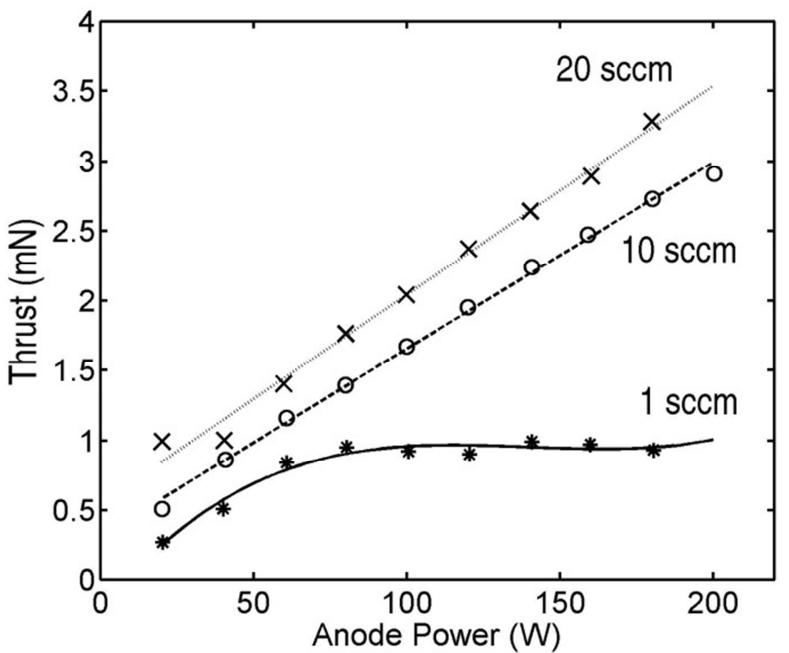

Figure 8. Thrust versus anode power at 1, 10, and 20 Figure 9. Specific impulse versus anode power at 1, 10, sccm flow rates.

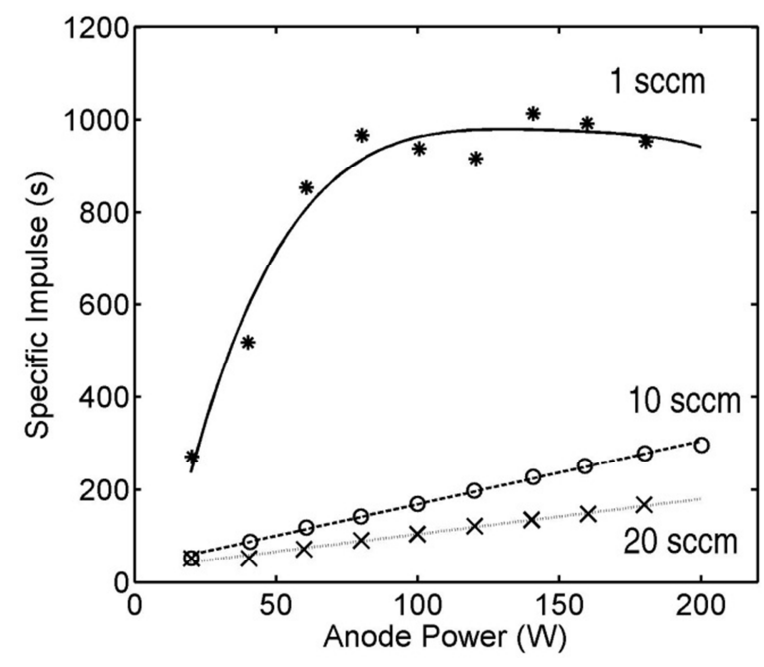

and $20 \mathrm{sccm}$ flow rates. 
The thrust and specific impulse of the device as a function of anode power are provided in Fig. 8 and 9 respectively. We can observe a difference in the behavior of the thruster between the high and low flow rate conditions. For the $1 \mathrm{sccm}$ flow rate the thrust plateaus, whereas for the $10 \mathrm{sccm}$ and $20 \mathrm{sccm}$ conditions the thrust continues to increase linearly up to $200 \mathrm{~W}$. Consequently, for higher levels of power we can achieve a significant increase in the level of thrust produced by moving to $10 \mathrm{sccm}$ of propellant flow. However, for flow rates higher than $10 \mathrm{sccm}$, the increase in thrust is far smaller: only a $17 \%$ increase in thrust is achieved at $200 \mathrm{~W}$ by increasing the propellant flow to $20 \mathrm{sccm}$. Examining the increase in thrust between the $10 \mathrm{sccm}$ and $20 \mathrm{sccm}$ conditions, we can see a roughly uniform increase of approximately $0.5 \mathrm{mN}$. This compares to a cold gas contribution from Xenon alone that is approximately $0.2 \mathrm{mN}$ per $10 \mathrm{sccm}$ of flow. Therefore, the increase in thrust may likely be accounted for by a hot gas expansion of the neutral Xenon propellant and points to the fact that the

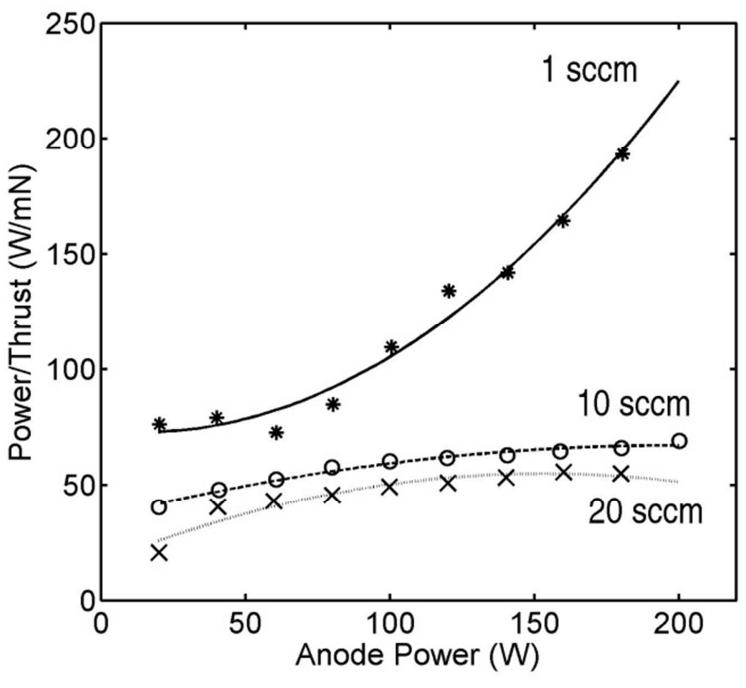

Figure 10. Power to thrust ratio versus anode power at 1,10 , and $20 \mathrm{sccm}$ flow rates. propellant is being underutilized in high flow regimes.

The trade-off between the thruster performance and propellant flow rate can be further examined by considering the power to thrust ratio for the three flow conditions (see Fig. 10). The improvement in the power to thrust ratio is significant when increasing the flow rate from 1 to $10 \mathrm{sccm}$ : at $100 \mathrm{~W}$ of anode power we can see an improvement of $45 \%$. However, when increasing the flow from $10 \mathrm{sccm}$ to $20 \mathrm{sccm}$ we can only achieve a further improvement of $18 \%$, which is likely outweighed by the relative decrease in the specific impulse.

\section{Conclusion}

The purpose of this study was to establish whether the QCT device could be run off-design, with higher levels of propellant flow, for occasions during satellite operation where the requirements for increased thrust outweigh the demand for effective propellant utilization. For comparison, we considered a nominal QCT operating point of 1000s specific impulse, $75 \mathrm{~W}$ of power, $1 \mathrm{mN}$ of thrust, and $1 \mathrm{sccm}$ of propellant flow.

The findings of this study clearly show that the performance of the QCT is highest with low propellant flow rates. However, some improvement to the thrust can be achieved by increasing the flow rate to $10 \mathrm{sccm}$. The thrust was found to increase from $1 \mathrm{mN}$ to $3 \mathrm{mN}$ at $200 \mathrm{~W}$ supplied power. Unfortunately, this gain in thrust is offset by a reduction in specific impulse from 1000s to 300s. A further increase to the flow from $10 \mathrm{sccm}$ to $20 \mathrm{sccm}$ resulted in only a marginal increase in the thrust (up to $3.3 \mathrm{mN}$ ), and a further decrease in the specific impulse (below 200s). It is likely that for these higher levels of flow the propellant is being underutilized, and the increased thrust can be attributed to a hot gas expansion of the neutral Xenon propellant rather than the production of additional accelerated ions.

\section{Acknowledgments}

This research has been funded through the University of Surrey's strategic partnership with EADS Astrium. The authors would like to thank the many people from Astrium who have contributed to this project.

\section{References}

${ }^{1}$ A. Knoll, U. Shafiq, and V. Lappas, "3-Dimensional Mapping of Plasma Properties in the Plume Region of the Quad Confinement Thruster", proceedings of the Space Propulsion 2012, Bordeaux, France, May 7 - 10, 2012.

${ }^{2}$ A. Knoll, B. Melly, V. Lappas, "The Quad Confinement Thruster - Preliminary Performance Characterization and Thrust Vector Control", Wiesbaden, Germany: 32nd International Electric Propulsion Conference, Spetember 11 - 15, 2011.

${ }^{3}$ M. Pollard, D. Lamprou, "The Design, Development, Manufacture and Test of an Electric Propulsion System for Small Spacecraft", proceedings of the Space Propulsion 2012, Bordeaux, France, May 7 -10, 2012.

${ }^{4}$ Pottinger, S. J., D. Lamprou, A. K. Knoll, and V. J. Lappas. "Impact of plasma noise on a direct thrust measurement system.” Review of Scientific Instruments 83, no. 3 (2012): 033504-033504. 